\title{
Mobility routing optimization for physical accessibility and thermoregulation
}

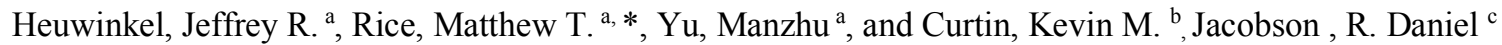 \\ ${ }^{a}$ Department of Geography and Geoinformation Science, George Mason University, Fairfax, Virginia, USA, email: jheuwink@, \\ rice@,myu7@gmu.edu \\ ${ }^{b}$ Department of Geography and Laboratory for Location Science, University of Alabama, Tuscaloosa, Alabama, USA, email: \\ kmcurtin@ua.edu \\ ${ }^{c}$ Department of Geography, University of Calgary, Alberta, Canada, email: dan.jacobson@ucalgary.ca \\ * Corresponding author
}

\begin{abstract}
:
As routing applications become common on mobile devices, significant problems that remain are the sparse underlying data support for pedestrian-based routing and the inability to customize an existing route for specific individual accessibility needs. Cartographic researchers have repeatedly demonstrated methods for sophisticated modelling of infrastructure and have built routing portals and accessibility systems, yet these systems and their benefits have not been used widely, due to problems with underlying data support. This research reviews a few exemplar systems and presents a new routing study that uses the presence of overhead tree canopy to add a preference layer to individual routing. This allows individuals to plan and choose navigation pathways for purposes of body heat thermoregulation, a problem that exists for many individuals with mobility impairments, particularly those with spinal cord injuries. The study presented here demonstrates that successful routing underneath the tree canopy can be done in a way that only marginally increases the length of such routes. This study also demonstrates the need for detailed geographic data support for preference-based routing.
\end{abstract}

Keywords: accessibility mapping, routing, optimization

\section{Introduction and background}

Interest in personalized routing applications has risen in the past decade, with the growth smart phones and the increasing sophistication of navigation applications. Research in this area has also been of interest to cartographers and geographic information scientists who seek to build better applications and provide data support for such applications. Until recently, most navigation applications and portals performed pedestrian routing using existing street networks, without knowledge of the presence of sidewalks, crosswalks, crossing signals, or other necessary infrastructure. While data support for true pedestrian routing is improving, the level of detail required for many routing tasks simply does not exist. Laakso et al. (2013) approaches this problem through formal modelling of information within supporting databases and by identifying routing requirements. Others, such as Beale et al. (2006) analyse the needs of individuals using wheelchairs and build a GIS-based application for routing that incorporates slope, surface type, and curb cuts. This echoes the general suggestion by Perkins (2002) who advocates for tactile mapping strategies that are usercentered and based on the social context of map use. Studying the needs of the end-users, especially the most vulnerable, is likely to result in a more successful approach than a process driven only by technology.
Motivated by this earlier work, and by previous experience with accessibility mapping (Rice et al. 2005, Golledge et al. 2006), researchers conducted a broad study of local user needs, and discovered two significant areas where data support for wayfinding and navigation was lacking (Rice et al. 2013). First, local individuals needed updated information about temporary obstructions to navigation pathways, which caused a detour from predictable, accessible routes. This information was not incorporated into any existing navigation system, and was very difficult to collect. Second, users sought ways to meet specific individual needs while navigating public space, such as the need to avoid areas with steep cross slope, which makes the steering and navigation of power wheel chairs difficult, especially for individuals without the ability to selfbalance. Data support for this second area of interest could only be met by very detailed and comprehensive data collection and modelling of infrastructure, e.g., as presented by Laakso et al. (2013).

Reviews of detailed personalized routing portals (Rice et al. 2014) found several examplars, including the Accessible Paths in Pinhel, which like Beale et al. (2006) incorporates surface texture, curb cuts, and cross walks. A second exemplar is the Federal Polytechnical School of Lausanne's campus map, which offers detailed indoor and outdoor navigation solutions to compensate for significant elevation changes and the presence of stairs and other 
inaccessible paths. Rice et al.'s review (2014) also identified a few new areas for research. Individuals with vision and mobility impairments were asked to indicate their normal routes across a college campus. When several subjects identified routes several times longer than the typical shortest distance route, they indicated that their preferred route was taken to avoid long exposure to direct sunlight, which led to problems with body thermoregulation during the relatively hot portions of the spring, summer, and fall. In support of this finding, Klenck and Gebke (2007) note that individuals with mobility impairment, especially those with spinal cord injury, often have difficulty regulating body temperature in hot and cold environments. Routing underneath the tree canopy or through buildings is a preferred way to help regulate body temperature, according to individuals studied. These individuals also noted that detouring around temporary obstacles within an otherwise dependable and accessible route was a significant problem. With this information, researchers pursued a dual effort to find ways to map these temporary obstacles, and to incorporate support for individual routing preferences such as the presence of an overhead tree canopy.

\section{Mapping transient obstacles through geocrowdsourcing}

A useful approach for mapping transient obstacles is geocrowdsourcing, which has fundamentally changed the way that geospatial data is collected and used (Sui et al. 2012). Geocrowdsourcing can be difficult to implement, and it requires a large population of contributors within a small area. The primary advantage is timeliness. Zook et al. (2010) and Goodchild and Glennon (2012) provide evidence that geocrowdsourcing can be very effective in dealing with rapidly unfolding events, such as natural disasters. The main drawback to the approach is reliability and accuracy, as reviewed helpfully by Senaratne et al. (2017), who cite an influential earlier review of accuracy assessment methods by Goodchild and $\mathrm{Li}$ (2012). Accuracy assessment of geocrowdsourced data can be difficult to perform, but Goodchild and $\mathrm{Li}$ present three fundamental strategies, all of which have been incorporated in this work.
The GMU Geocrowdsourcing Testbed (Qin et al. 2015, Rice et al. 2014, Rice et al. 2018) traces the trajectory of geocrowdsourcing techniques for mapping transient obstacles. It initially incorporated a desktop-based application (Figure 1) which used social moderation approach for accuracy assessment (Rice et al. 2014). The same system later added mobile applications (Figure 2), and incorporated additional quality control methods (Rice et al. 2015).

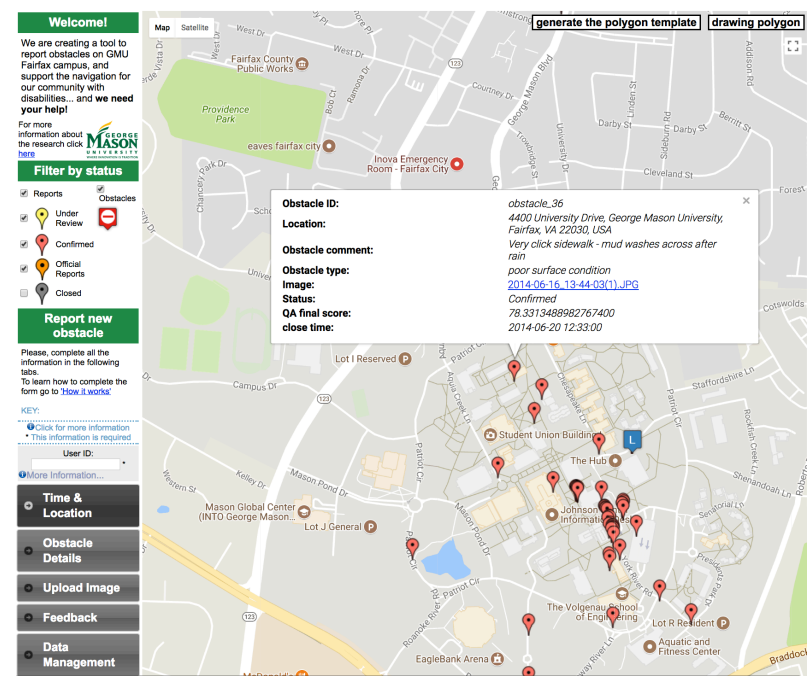

Figure 1. The GMU Geocrowdsourcing Testbed, desktop implementation

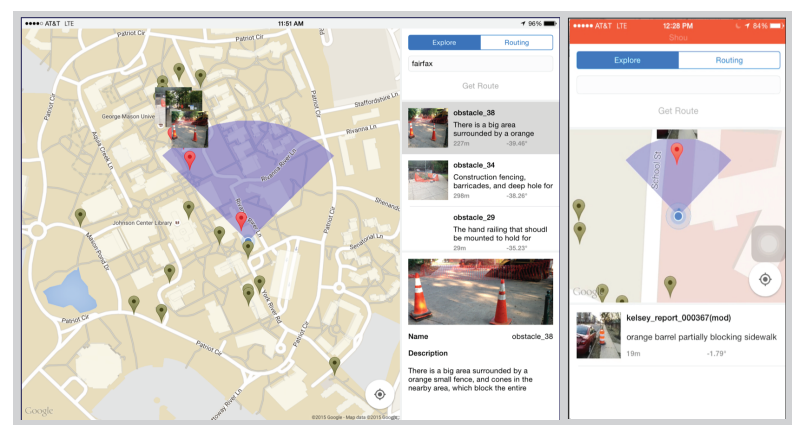

Figure 2: GMU Geocrowdsourcing Testbed, mobile tablet (left) and smart phone applications with navigation avoidance capabilities

The GMU Geocrowdsourcing Testbed's crowdsourcing quality assessment approach was demonstrated by Williams (2018) to have the same level of accuracy as the earlier resource intensive social moderation approach. New accuracy assessment metrics and methods are based on mobile device positioning dynamics, EXIF data from images, and geocrowdsourcing contribution dynamics (Figure 3, Rice et al. 2018). 

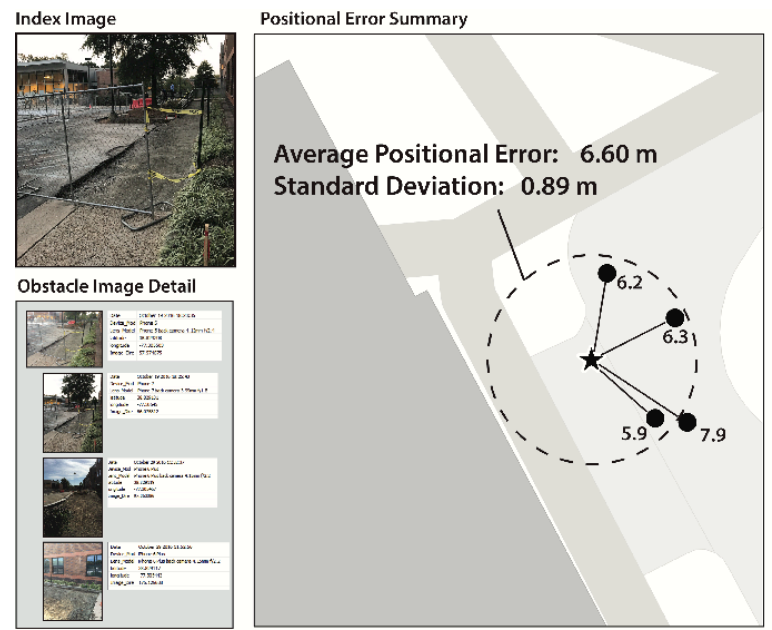

Figure 3. Positional error derived from mobile positioning dynamics and EXIF data in the new GMU Geocrowdsourcing Testbed (Rice et al. 2018)

\section{Tree canopy preference routing}

In the earlier individual accessible routing preference study, several individuals with visual and mobility impairments mapped their individual routing preferences. Stairway and steep running slope avoidance were common preferences, as were preferences for signalized crossing points and pathways with curb cuts. Qin et al. (2015) studied these preferences and developed statistics for route deviation from shortest distance route. Routes with avoidance for stairs, steep paths, and temporary obstacles identified through geocrowdsourcing were between six percent longer, and in extreme cases, two thousand percent longer. One distinct routing preference stated by individuals with mobility impairments, was routing that incorporated tree canopy coverage. This was described as a way to regulate body heat. Routing underneath the tree canopy during the warmer eight months of the year, as well as routing through air-conditioned buildings, was a preference that was thought to be easily incorporated into local navigation and routing portals. Subsequently, a routing study was conducted to assess the feasibility of incorporating this preference, and to analyze the relative increase in route length, compared the shortest distance route.

For this study, the first step was to map the tree canopy.This was done for the local area through vectorbased digitizing using high-resolution imagery. Next, the local pedestrian network was densified and given a value to indicate the presence of tree canopy directly overhead. A routing algorithm was developed in Esri's ArcGIS to compute a route cost using the normal segment length methods, resulting in a shortest distance route. This was then compared with an algorithm where the segments with canopy coverage received a significantly lower cost. With some adjustment and testing, cost functions related to canopy and length were developed to identify routes with a similar length but with significantly lower costs. Figures 4,5 , and 6 show the results of this work, using three of the six examples route selected. Figure 4 shows a typical result, where the canopy coverage of a route (in red) can be increased significant (47\%) with an associated 32\% increase in length. This represents a typical route presented by study participants. Figures 5 and 6 show a more dramatic result, where a significant increase in canopy coverage ( $48 \%$ and $50 \%)$ are achieved with routes that are only slightly longer $(9.8 \%$ and $2.8 \%)$.

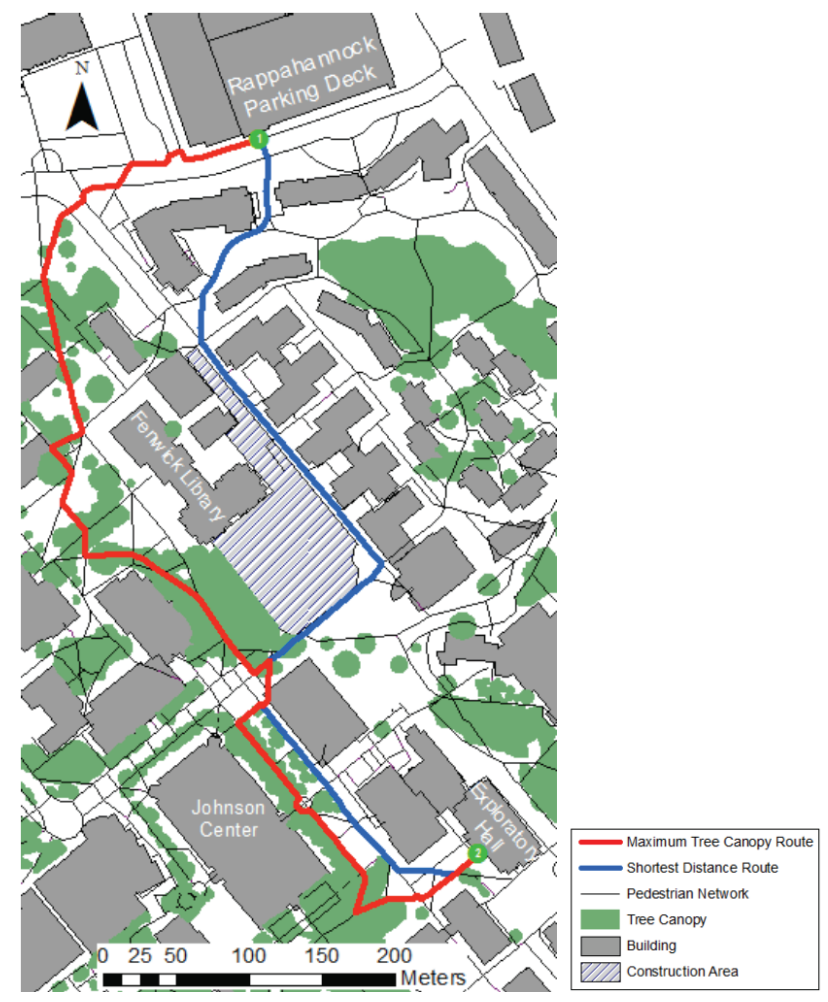

Figure 4. Red tree-canopy maximizing route results in a $47 \%$ increase in canopy coverage and $32 \%$ increase in length.

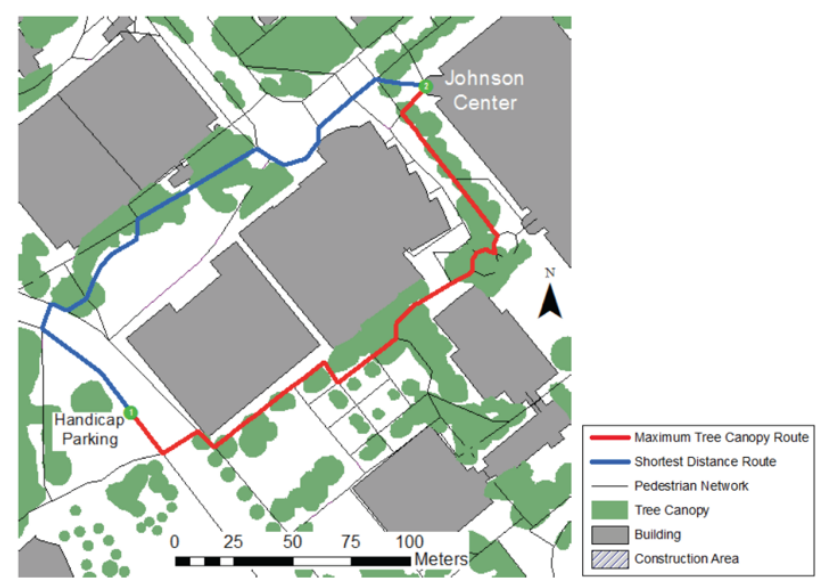

Figure 5. Red tree-canopy maximizing route results in a $48 \%$ increase in canopy coverage and a $9.8 \%$ increase in length relative to the blue shortest distance route. 


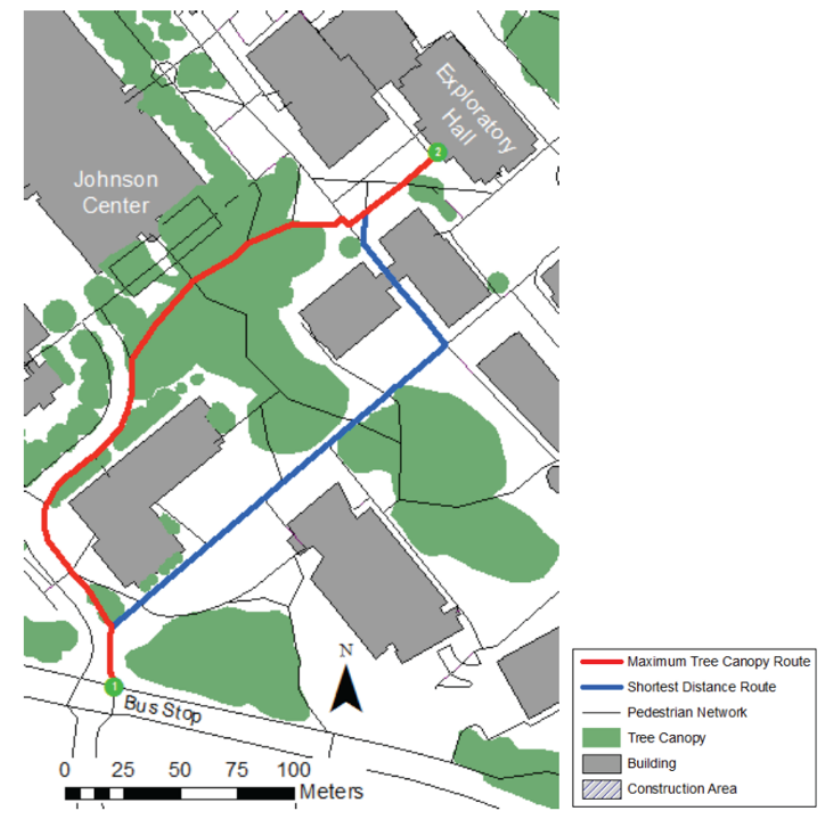

Figure 6. Red tree-canopy maximizing route results in a 50\% increase in canopy coverage with a $2.8 \%$ increase in length.

While variables such as sun angle, time of day, time of year, latitude, and building shadow are not analysed in this work, maximum tree canopy coverage routes significantly increase the tree canopy coverage percentage without increasing route length. These variables are planned for implementation during the next phase of development, and may significantly improve the ability to develop preference-based accessible routing.

\section{Conclusions}

Developing preference-based accessible routing requires significant underlying data to support the workflows. Laakso et al. (2013) and others have demonstrated modelbased approaches, and data requirements for working at detailed scales and in settings where detailed individual accessible routing needs can be met. Two specific needs identified in this work are the ability to identify temporary obstacles in a travel path and route around them, and the ability to select routes with specific preference criteria, such as the presence of tree canopy. This tree canopy preference is similar to the preference for interior routing, and helps end-users preserve or shed body heat, depending on the season. This work demonstrates that with standard GIS software, routes can be identified which are only slightly longer, but result in a significant increase in canopy coverage. While some variables such as time of day, season, local latitude, and building shadow are not incorporated in this routing analysis, adding those variables is the next step. The variables, along with tree canopy data, can be readily derived from common sources, including LiDAR. The identification of temporary or transient obstacles, is more challenging and requires a specific geocrowdsourcing framework, and a significant body of dedicated contributors. This temporary obstacle information can be combined with other well-known and non-transient accessibility hazards (stairs, steep pathways, poor surface conditions, absence of curb cuts, absence of signalization) to produce accessible routes. The work on this project has been enhanced greatly by cartographic research presented at past ICC meetings, and will continue through the future.

\section{References}

Beale, L., Field, K., Briggs, D., Picton, P., \& Matthews, H. (2006). Mapping for wheelchair users: Route navigation in urban spaces. The Cartographic Journal, 43(1), 68-81.

Golledge, R. G., Rice, M., \& Jacobson, R. D. (2005). A commentary on the use of touch for accessing on-screen spatial representations: The process of experiencing haptic maps and graphics. The Professional Geographer, 57(3), 339-349.

Goodchild, M. F., \& Glennon, J. A. (2010). Crowdsourcing geographic information for disaster response: a research frontier. International Journal of Digital Earth, 3(3), 231-241.

Goodchild, M. F., \& Li, L. (2012). Assuring the quality of volunteered geographic information. Spatial statistics, 1 , 110-120.

Klenck, C., \& Gebke, K. (2007). Practical management: common medical problems in disabled athletes. Clinical Journal of Sport Medicine, 17(1), 55-60.

Laakso, M., Sarjakoski, T., Lehto, L., \& Sarjakoski, L. T. (2013). An information model for pedestrian routing and navigation databases supporting universal accessibility. Cartographica: The International Journal for Geographic Information and Geovisualization, 48(2), 89-99.

Perkins, C. (2002). Cartography: progress in tactile mapping. Progress in Human Geography, 26(4), 521530.

Qin, H., Rice, R. M., Fuhrmann, S., Rice, M. T., Curtin, K. M., \& Ong, E. (2016). Geocrowdsourcing and accessibility for dynamic environments. GeoJournal, 81(5), 699-716.

Rice, M., Jacobson, R. D., Golledge, R. G., \& Jones, D. (2005). Design considerations for haptic and auditory map interfaces. Cartography and Geographic Information Science, 32(4), 381-391.

Rice, M. T., Curtin, K. M., Paez, F. I., Seitz, C. R., \& Qin, H. (2013). Crowdsourcing to support navigation for the disabled: A report on the motivations, design, creation and assessment of a Testbed environment for accessibility (Annual No. BAA:\# AA10-4733, Contract:\# W9132V-11-P-0011). George Mason University, Fairfax, Virginia, United States.

Rice, M. T., Paez, F. I., Rice, R. M., Ong, E. W., Qin, H., Seitz, C. R., \& Medina, R. M. (2014). Quality assessment and accessibility applications of crowdsourced geospatial data: A report on the development and extension of the George Mason University Geocrowdsourcing Testbed. (Annual No. BAA:\# AA104733, Contract:\# W9132V-11-P-0011)(p. 91). George Mason University, Fairfax, Virginia, United States.

Rice, M. T., Curtin, K. M., Pfoser, D., Rice, R. M., Fuhrmann, S., Qin, H., ... \& Seitz, C. R. (2015). Social 
moderation and dynamic elements in crowdsourced geospatial data: A report on quality assessment, dynamic extensions and mobile device engagement in the George Mason University Geocrowdsourcing Testbed. (Annual No. BAA:\# AA10-4733, Contract:\# W9132V-11-P0011) George Mason University, Fairfax, Virginia, United States.

Rice, M. T., Jacobson, D., Pfoser, D., Curtin, K. M., Qin, H., Coll, K., ... \& Aburizaiza, A. O. (2018). Quality Assessment and Accessibility Mapping in an ImageBased Geocrowdsourcing Testbed. Cartographica: The International Journal for Geographic Information and Geovisualization, 53(1), 1-14.

Senaratne, H., Mobasheri, A., Ali, A. L., Capineri, C., \& Haklay, M. (2017). A review of volunteered geographic information quality assessment methods. International Journal of Geographical Information Science, 31(1), 139-167.

Sui, D., Elwood, S., \& Goodchild, M. (Eds.). (2012). Crowdsourcing geographic knowledge: volunteered geographic information (VGI) in theory and practice. Springer Science \& Business Media.

Williams, T. J. (2018). Mobile Positioning Dynamics in an Image-Based Hybrid Geocrowdsourcing System (Master of Science Thesis, July 2018) Fairfax, Virginia USA: George Mason University.

Zook, M., Graham, M., Shelton, T., \& Gorman, S. (2010). Volunteered geographic information and crowdsourcing disaster relief: a case study of the Haitian earthquake. World Medical \& Health Policy, 2(2), 7-33. 